\title{
Anatomical relation of third molars and the retromolar canal
}

\author{
C. Moreno Rabie ${ }^{a, b}$, M. Vranckx ${ }^{a, *}$, M.I. Rusque ${ }^{a, b}$, C. Deambrosi ${ }^{\text {a }}$, \\ A. Ockerman ${ }^{\text {a }}$, C. Politis ${ }^{\text {a }}$, R. Jacobs ${ }^{\mathrm{a}, \mathrm{c}}$ \\ ${ }^{a}$ OMFS-IMPATH Research Group, Department of Imaging and Pathology, Faculty of Medicine, University of Leuven, and Department of Oral and \\ Maxillofacial Surgery, University Hospitals Leuven, Leuven, Belgium \\ ${ }^{\mathrm{b}}$ Department of Oral and Maxillofacial Radiology, Universidad de los Andes, Santiago, Chile \\ ${ }^{c}$ Department of Dental Medicine, Karolinska Institutet, Stockholm, Sweden
}

Accepted 7 July 2019

\begin{abstract}
The study was designed to assess, by direct anatomical observations and cone-beam computed tomography (CT), the prevalence of a retromolar canal and foramen in relation to the mandibular third molar in dry human mandibles. Dry mandibles from European skeletons $(\mathrm{n}=89) \mathrm{were}$ observed directly and after cone-beam CT scanning (Newtom VGI evo). The following variables were assessed: the presence of a retromolar foramen and canal; the presence of a third molar, and orientation of the third molar. From the total of 89 mandibles selected, 73 showed a retromolar foramen (49 of which were bilateral). A retromolar canal was identified in 64 mandibles based on cone-beam CT assessment, with a total of 101 canals, including 74 that were bilateral. A total of 112 hemimandibles contained a third molar. Orientation of the third molar did not seem to indicate the presence of retromolar foramina or canals. A similar prevalence of retromolar canals was found for both vertically (41/79) and mesially (17/33) orientated third molars.

A retromolar foramen and canal were present in most mandibles, with more than half being bilateral. We were unable to confirm a potential relation between the retromolar foramen and canal on the one hand, and the orientation of the third molar on the other.

(C) 2019 The British Association of Oral and Maxillofacial Surgeons. Published by Elsevier Ltd. All rights reserved.
\end{abstract}

Keywords: Cone-beam CT; retromolar canal; retromolar foramen; third molar; wisdom tooth

\section{Introduction}

Distal to the third mandibular molar is a triangular area, better known as the retromolar area, delimited laterally by the buccinator muscle, mesially and posteriorly by the tendon of the temporal muscle, and anteriorly by the alveolar process

\footnotetext{
* Corresponding author at: OMFS-IMPATH research group KU Leuven Kapucijnenvoer 333000 Leuven Belgium. Tel.: +3216341975

E-mail addresses: cmoreno@miuandes.cl (C. Moreno Rabie), myrthel.vranckx@kuleuven.be (M. Vranckx), mirusque@miuandes.cl (M.I. Rusque), deambrosicamilla@gmail.com (C. Deambrosi), anna.ockerman@kuleuven.be (A. Ockerman), constantinus.politis@uzleuven.be (C. Politis), reinhilde.jacobs@uzleuven.be (R. Jacobs).
}

of the third molar. Some variations can be seen in this zone, such as the presence of a foramen that can be the output of a canal containing a neurovascular bundle. ${ }^{1}$

The presence of a retromolar canal was first described by Schejtman et al in 1967 and then by Ossenberg in $1987 .^{1,2} \mathrm{It}$ can be defined as a branch of the inferior alveolar neurovascular bundle that runs along a bony path from the mandibular canal to its exit through the retromolar foramen., ${ }^{1,2}$ The canal has been reported to contain a portion of nerves and at least one artery and vein, in which its clinical importance lies. ${ }^{1}$ It has been associated with complications during third molar extractions or procedures in the retromolar area such as failure of an anaesthetic block, haemorrhage, and iatrogenic 
nerve damage. ${ }^{2-5}$ Surgeons operating in this area should consider the presence and width of the canal preoperatively.

The retromolar canal can be identified radiographically. Unfortunately, most of the time conventional 2-dimensional radiographs such as panoramic radiographs cannot identify variants of these anatomical structures because of inherent technical characteristics, such as the tomographic effect, 2-dimensional anatomical superposition, distortion and magnification of the image, and low spatial resolution. ${ }^{5,6}$ Cone-beam computed tomography (CT) can identify it more accurately, considering its three dimensional nature. ${ }^{7,8}$

Various authors have reported considerable differences in the prevalence of retromolar canals and foramina, with numbers ranging from $12 \%$ to $75 \%$ for canals and 0 to $52 \%$ for foramina. ${ }^{1,5,7,9-16}$ These results can be explained by the study sample, design (in vivo or ex vivo), radiographic technique (2- compared with 3-dimensional), inclusion and exclusion criteria for one-beam CT compared with medical CT (better trabecular delineation), and the anatomical definition used.

The relation of the retromolar canal to the third molar has not been described to our knowledge, except for complications with the neurovascular bundle during extraction of third molars or other oral and maxillofacial procedures in the retromolar area. ${ }^{2-5}$ We know of no information about orientation of third molars or type of canal, its presence or absence, or the association of the appearance of a retromolar canal and the gubernacular cord of the third molar.

The aim of the present study was to assess the prevalence of retromolar canals and foramina in relation to the mandibular third molar in dry human mandibles by direct anatomical observations and cone-beam CT imaging.

\section{Material and methods}

\section{Sample}

The initial sample consisted of 183 dry human mandibles of European origin, age and sex unknown. Ethics approval was obtained from the Medical Ethics Committee of the University Hospitals of Leuven (Belgium). Eighty-nine mandibles were included for further analysis.

\section{Macroscopic evaluation}

The sample was classified macroscopically according to the unilateral or bilateral presence, and the eruption status, of third molars (eruption, impaction, agenesis or missing, either from extraction or post mortem loss) and unilateral or bilateral presence or absence of a retromolar foramen. To consider the perforation in the retromolar area as a foramen, it was measured empirically and reported as present or not present in each hemimandible (Fig. 1).

\section{Radiographic evaluation on cone-beam CT}

Mandibles were scanned with a cone-beam CT (Newtom VGI evo,) with soft tissue substitution ( $\mathrm{Cu}$ filter). The height of the field of view was $10 \mathrm{~cm} \times 5 \mathrm{~cm}$ with a pixel value of $0.125 \mathrm{~mm}$. Separately from, and independently of, direct anatomical observations (macroscopic screening), each mandible was radiographically evaluated on conebeam CT images for the presence of a retromolar canal by viewing the continuous sectional images using MeVisLab software. To consider the presence or absence of the retromolar canal, the radiographic definition given by Capote et al was used: "The identification of a radiolucent image bounded by radiopaque lines present in the retromolar triangle, associated or not with the distal inferior tooth and/or with the mandibular canal". ${ }^{5}$ Any canal that met this definition, regardless of its width, was considered as retromolar.

The orientation of third molars was assessed to search for a relation between the presence of a retromolar canal and the position of the third molar in the mandible. Threedimensional reconstruction of the imaging data was made for this assessment. The following classification was used: not present, (functional) vertical axis orientation, and mesio-

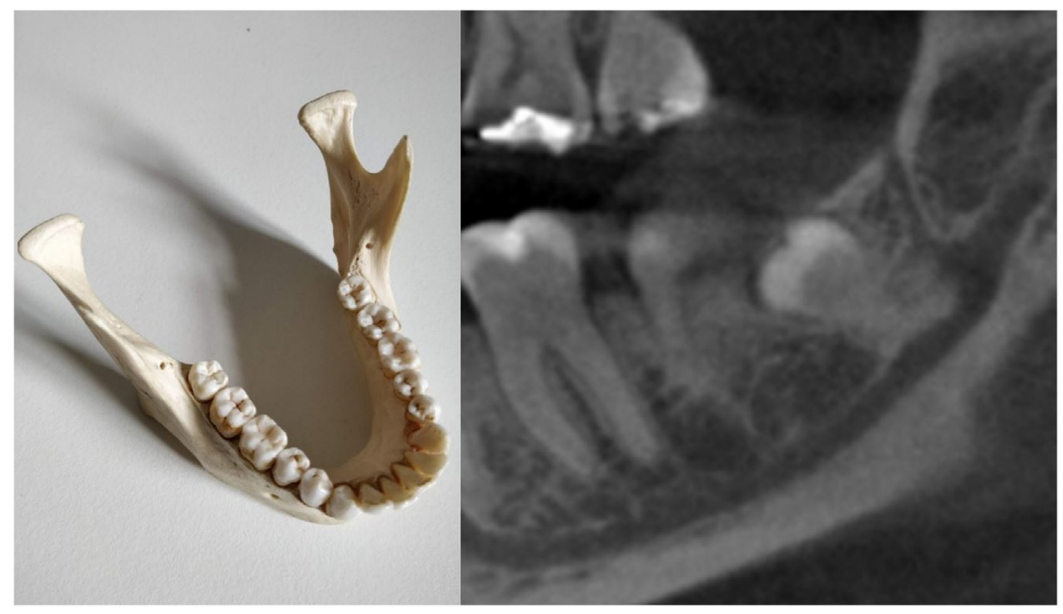

Fig. 1. Macroscopic observation of the retromolar foramen and sagittal view of the retromolar canal on cone-beam computed tomography. Images from own database. 


\section{Presence of RMF macroscopically}

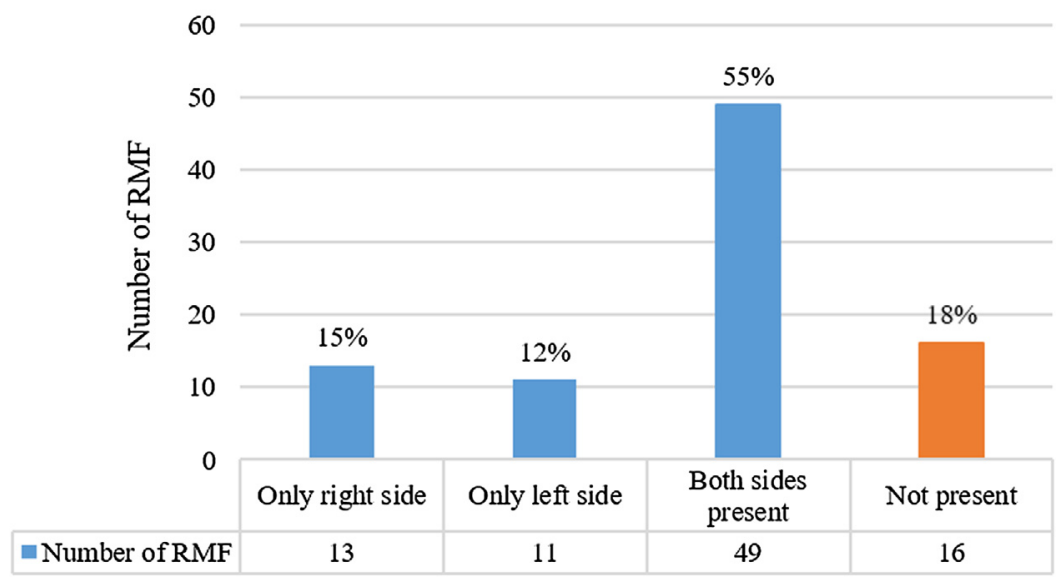

Fig. 2. Presence of retromolar foramina and side distribution in a total of 89 mandibles observed macroscopically. RMF = retromolar foramen.

and distoangulation of the third molar. Lastly, the number of teeth present in each hemimandible was also noted. Descriptive statistics were used for all variables assessed during macroscopic and radiographic evaluation.

\section{Results}

\section{Presence of retromolar foramina macroscopically}

Of a total of 89 mandibles, 73 showed the presence of unilateral or bilateral foramina on macroscopic evaluation, with 49 showing bilateral foramina. Thirteen mandibles showed a foramen only on the right side and 11 only on the left side. The total number of retromolar foramina was 122 , located in 73 mandibles (Fig. 2).

\section{Presence of retromolar canals during radiographic evaluation}

Of 89 mandibles, 64 showed a retromolar canal unilaterally or bilaterally during cone-beam CT assessment, and the total was 101 . Seventy-four were bilateral and 27 unilateral. From the latter, 20 were on the right and 7 on the left, giving a total of 57 canals on the right and 44 on the left (Fig. 3).

Forty-nine foramina turned into a retromolar canal on the right and 34 on the left. Both a foramen and a canal were absent in 19 on each side. This means that macroscopic and radiographic observations in 57 hemimandibles did not match, either because the macroscopic evaluation showed a foramen and the cone-beam CT did not, or vice versa.

\section{Relation between the orientation of the third molar and the retromolar canal}

One hundred and twelve hemimandibles contained a third molar. The sample consisted of 58 hemimandibles with third molars and retromolar canals, and 54 that had a third molar but showed no sign of a retromolar canal (Fig. 4).

The canal was most likely to be present if the third molar was orientated on the vertical axis. So far the data showed no relation between the presence of the retromolar canal and the orientation of the third molar (Fig. 4), which seems not to indicate the presence of a retromolar canal. A similar prevalence of canals was found for both vertically (41/79) and mesially (17/33) orientated third molars.

\section{Discussion}

The retromolar trigone and its anatomical variations such as the retromolar foramen and retromolar canal can be important when it comes to the extraction of third molars, treating mandibular fractures, and planning orthognathic surgery or any other procedure in this area. Local complications have been reported, including anesthetic failure ${ }^{2,3,10,15,17}$ and haemorrhage, ${ }^{3,17}$ and neurosensory disturbances in the retromolar area ${ }^{3,10,17}$ such as paraesthesia after the extraction of a lower third molar, as reported by Singh. ${ }^{18}$ That patient experienced numbness of the gingiva from the buccal sulcus to the canine when compared with the contralateral side. ${ }^{18}$

The present study aimed to assess the prevalence of retromolar foramina and canals in dry mandibles, and to evaluate the potential relations of the presence of a retromolar canal with the orientation of the ipsilateral third molar.

The high prevalence of retromolar foramina during macroscopic observation can be attributed to the inclusion of all foramina in the triangle, irrespective of their diameter. As far as retromolar canals are concerned, the results show that at least one was present in 64 of the mandibles, totalling 101 of the 178 hemimandibles examined. Even the smallest diameter $(<1 \mathrm{~mm})$ was considered. De Oliveira-Santos et al reported that the width of the canal should be at least $2 \mathrm{~mm}$ to be clinically relevant. ${ }^{19}$ Canals with a diameter $<2 \mathrm{~mm}$ are 


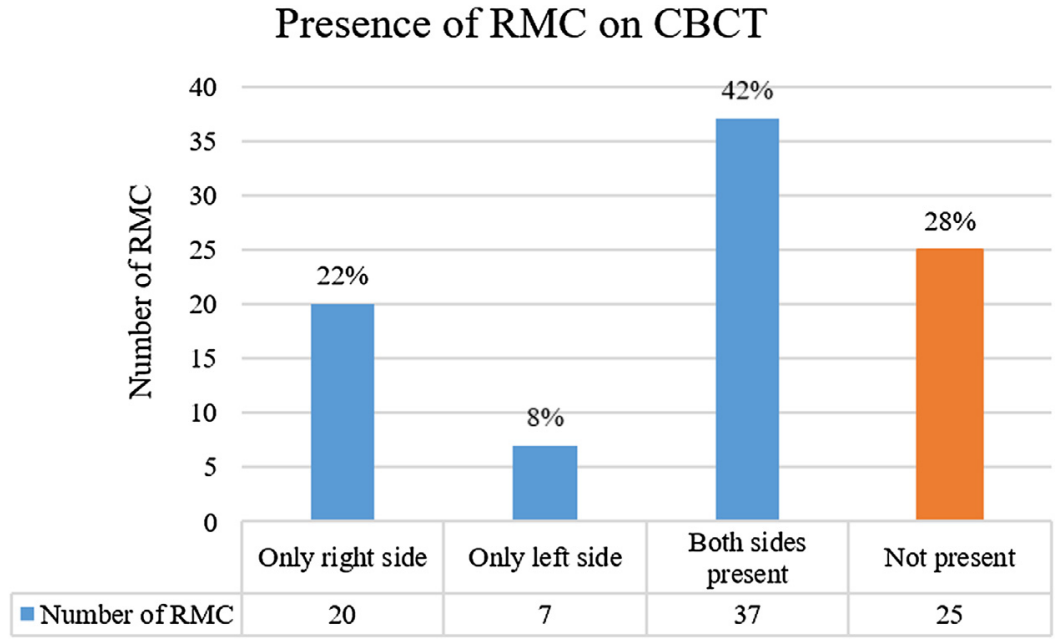

Fig. 3. Presence of retromolar canals found on cone-beam computed tomography in a total of 89 mandibles. RMC $=$ retromolar canal.

\section{Third molar orientation and presence of the RMC}

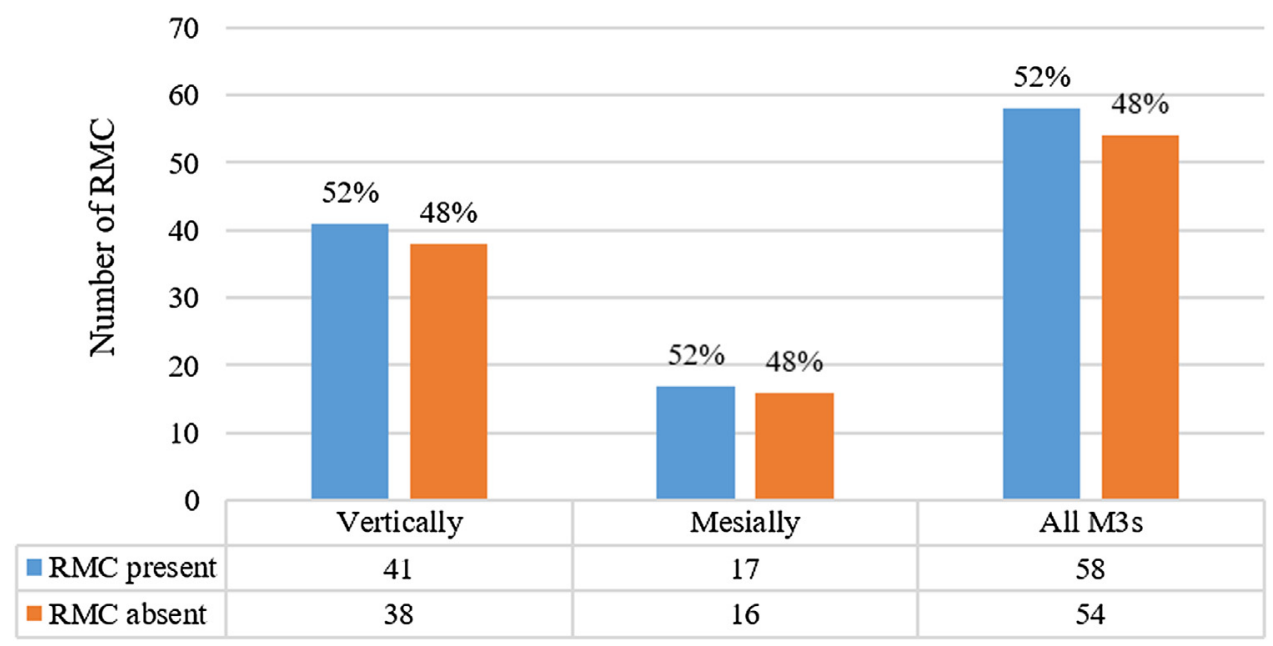

Fig. 4. Presence of the retromolar canal according to orientation of the third molar. Analysis of 112 hemimandibles with third molar present. RMC $=$ retromolar canal.

mostly considered irrelevant, even if they had a neurovascular content. This was considered by Gamieldien and Van Schoor, who reported a prevalence of only $8 \%$ of retromolar foramina in 885 dry mandibles. ${ }^{4}$ They considered a foramen to be "any perforation in the retromolar area that allowed the passage without resistance of a needle of $1 \mathrm{~mm}$ in diameter". The reason for not considering a smaller diameter is that a smaller calibre canal would contain a smaller neurovascular bundle and its clinical complications would not be relevant. ${ }^{4}$ It is possible that for this reason the evidence for complications reported to be associated with retromolar canals is contradictory. Gameldien and Van Schoor concluded that there is limited evidence that the retromolar foramina and canals are responsible for anaesthetic failure. Additional bleeding during procedures may occur in this area, but can easily be controlled. ${ }^{4}$
We would like to stress that our sample is $<100$, so that the reported percentages in the Figures are to be considered carefully, though reporting of these percentages is necessary to be able to compare our present study with existing publications. The reported prevalence of the retromolar canal and foramen ranged from 1.7 to $25 \%, 3,20$ varying among samples for type and age. Studies of cadaveric mandibles usually concentrate on the retromolar foramen rather than the canal. Athavale et al included dry ossified mandibles and adult cadavers, but excluded edentulous mandibles and the ones from which third molars had been extracted or had a resorbed third molar socket. ${ }^{9}$ The foramen was found in 10 mandibles out of 71 studied, and it was located $1 \mathrm{~cm}$ from the posterior border of the wisdom tooth. Kawai et al established the presence by counting the number of foramina present on each side $(27 \%){ }^{14}$ 

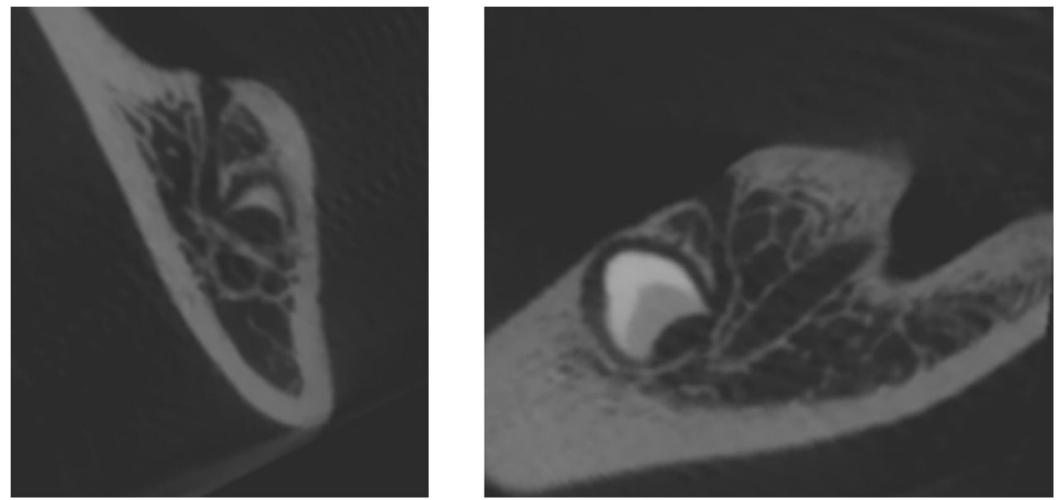

Fig. 5. Coronal (left) and sagittal (right) view of a dry mandible showing the presence of a large retromolar canal. The relation with the third molar follicle can clearly be seen. Images from own database.

Controversially Kawai et al found a relatively high prevalence $(52 \%)$ of retromolar foramina, but a similar number to us. ${ }^{14}$ These results can be explained by the different methods of observation. Ninety sides of 46 cadaveric mandibles of unknown sex were evaluated on cone-beam CT by a specialist observer who moved this plane mesiodistally and buccolingually to confirm the presence of the retromolar canal and foramen. The retromolar foramina were located lingually from the mandibular canal, with a prevalence of $52 \%$. This seems to confirm that it is not an unusual anatomical structure. The same conclusion was found in a retrospective study by Sisman et al. ${ }^{16}$

Schejtman et al also followed a similar protocol to ours and had corresponding findings. They studied and dissected 18 heads, of which $72 \%$ showed a single retromolar canal, and $27 \%$ bilateral ones. ${ }^{1}$ They confirmed that these canals arose from the mandibular canal and in many cases contained a neurovascular bundle branch. However, they did not record the width of the canal and foramen. Another study was conducted by Patil et al, who described retromolar canals in $75 \% .^{12}$ They were all seen in cone-beam CT images and all of them, irrespective of their diameter, were included.

The prevalence of retromolar foramina and canals found in groups of patients is generally lower than in dry mandibles. Lizio et al used a sample of 233 cone-beam CT images from 187 patients with a mean age of 46 years. ${ }^{7}$ The low prevalence (16\%) that they found, as also in the paper by Von Arx et al $(26 \%)$, can be explained by the restricted inclusion criteria chosen for the retromolar foramina (minimal diameter). ${ }^{15}$ These limits were set based on previous studies that considered only widths between $1.5-4.4 \mathrm{~mm}$ as being clinically relevant. ${ }^{10}$ On the other hand, Kang et al analysed cone-beam CT images from patients with an age range from 16 to 57 years old, and discovered that $53 \%$ of the bifurcations in the mandibular canal were retromolar canals. ${ }^{21}$

It is widely agreed that cone-beam CT is the examination method of choice for identifying retromolar canals. Because of their limited width it is difficult to detect the presence of accessory canals on panoramic radiographs. In addition, the superimposition of anatomical structures in this technique might hamper the observation of the canal. Von Arx et al found 31 canals in 121 sides using cone-beam CT, where only 7 of these canals could be seen on panoramic radiographs. ${ }^{15}$ Fukami et al reported that spiral CT was not effective for the assessment of this structure either, because the discrimination between the trabecular bone and the canal was not clear. ${ }^{22}$

Most of the research into retromolar foramina and canals is about incidence, characteristics of anatomical variations, and the clinical implications of their presence. In this study we have tried to evaluate the indicative value of the orientation of the third molars to the presence of a retromolar canal. Fig. 5 shows a dry mandible with a large retromolar canal, and although the relation with the third molar follicle is clearly seen, no statistical relation could be deduced from the present data.

Another interesting topic of research is the link between the canal that emerges through the retromolar foramen and the gubernaculum cord. This might be the reason why some authors described the distance between the foramen and the lower third molar. ${ }^{4,12}$ The gubernacular cord is a structure of connective tissue surrounded by a bony wall known as the gubernacular canal. ${ }^{23}$ This structure was first described by John Hunter in 1778 as a canal that connects tooth in formation with gums. ${ }^{24}$ It is said that the cord is derived from the fragmented remains of the dental lamina and contains connective tissue that includes nervous, vascular, and lymphatic portions, as well as epithelial remains. ${ }^{25}$ Cahill and Marks studied the role of the gubernaculum during tooth eruption. Despite the small sample collected, they concluded that this structure could have a role in the process, but its absence does not prevent it from happening. ${ }^{26}$

If the retromolar canal is a histological vestige of the gubernacular canal of the third molar, we should probably have found a higher incidence of this canal in relation to third molars with dental malpositions such as mesioangulation, or impacted or rotated teeth. More histological and morphological studies will be necessary to verify this hypothesis, as well as to assess the specific role of the gubernaculum in relation to orientation of wisdom teeth and the appearance of a retromolar canal. 
In this study, the prevalance of the retromolar canal and the structures within were higher than in previous studies. We conclude that this anatomical structure is more common than previously thought, but a potential relation between the presence of the retromolar canal and orientation of the third molar or its gubernaculum cord could not be confirmed in the sample. In any case, it should be considered when surgical procedures are needed in the retromolar area to avoid the occurrence of intraoperative and postoperative complications such as bleeding and neurosensory disturbances, most importantly when they have a wider diameter. Further studies are needed to assess the prevalence of retromolar foramina and canals, and to consider their diameter and clinical relevance.

\section{Conflict of interest}

We have no conflicts of interest.

\section{Ethics statement/confirmation of patients' permission}

Ethics approval was obtained from the Medical Ethics Committee of the University Hospitals of Leuven (Belgium). Patients' permission was not required.

\section{Acknowledgements}

We would like to thank Prof. Dr. Ivo Lambrichts (UHasselt) for putting his collection of dry mandibles at the disposal of our researchers.

\section{References}

1. Schejtman R, Devoto FC, Arias NH. The origin and distribution of the elements of the human mandibular retromolar canal. Arch Oral Biol 1967; 12:1261-7.

2. Ossenberg NS. Retromolar foramen of the human mandible. Am J Phys Anthropol 1987;73:119-28.

3. Bilecenoglu B, Tuncer N. Clinical and anatomical study of retromolar foramen and canal. J Oral Maxillofac Surg 2006;64:1493-7.

4. Gamieldien MY, Van Schoor A. Retromolar foramen: an anatomical study with clinical considerations. Br J Oral Maxillofac Surg 2016;54:784-7.

5. Capote TS, Gonçalves Mde A, Campos JÁ. Retromolar canal associated with age, side, sex, bifid mandibular canal, and accessory mental foramen in panoramic radiographs of Brazilians. Anat Res Int 2015;2015:434083.

6. White SC, Pharoah MJ. Oral radiology: principles and interpretation. 7th ed Mosby; 2014.
7. Lizio G, Pelliccioni GA, Ghigi G, et al. Radiographic assessment of the mandibular retromolar canal using cone-beam computed tomography. Acta Odontol Scand 2013;71:650-5.

8. Kaufman E, Serman NJ, Wang PD. Bilateral mandibular accessory foramina and canals: a case report and review of the literature. Dentomaxillofac Radiol 2000;29:170-5.

9. Athavale SA, Vijaywargia M, Deopujari R, et al. Bony and cadaveric Study of retromolar region. People J Sci Res 2013;6:14-8.

10. Narayana K, Nayak U, Ahmed W, et al. The retromolar foramen and canal in south Indian dry mandibles. Eur J Anat 2002;6:141-6.

11. Rossi AC, Freire AR, Prado GB, et al. Incidence of retromolar foramen in human mandibles: ethnic and clinical sspects. Int $J$ Morphol 2012;30:1074-8.

12. Patil S, Matsuda Y, Nakajima K, et al. Retromolar canals as observed on cone-beam computed tomography: their incidence, course, and characteristics. Oral Surg Oral Med Oral Pathol Oral Radiol 2013;115:692-9.

13. Orhan AI, Orhan K, Aksoy S, et al. Evaluation of perimandibular neurovascularization with accessory mental foramina using cone-beam computed tomography in children. J Craniofac Surg 2013;24:e365-9.

14. Kawai T, Asaumi R, Sato I, et al. Observation of the retromolar foramen and canal of the mandible: a CBCT and macroscopic study. Oral Radiol 2012;28:10-4.

15. von Arx T, Hänni A, Sendi P, et al. Radiographic study of the mandibular retromolar canal: an anatomic structure with clinical importance. J Endod 2011;37:1630-5.

16. Sisman Y, Ercan-Sekerci A, Payveren-Arıkan M, et al. Diagnostic accuracy of cone-beam CT compared with panoramic images in predicting retromolar canal during extraction of impacted mandibular third molars. Med Oral Patol Oral Cir Bucal 2015;20:e74-81.

17. Wyatt WM. Accessory mandibular canal: literature review and presentation of an additional variant. Quintessence Int 1985;1996(27):111-3.

18. Singh S. Aberrant buccal nerve encountered at third molar surgery. Oral Surg Oral Med Oral Pathol 1981;52:142.

19. de Oliveira-Santos C, Souza PH, de Azambuja Berti-Couto S, et al. Assessment of variations of the mandibular canal through cone beam computed tomography. Clin Oral Investig 2012;16:387-93.

20. Ossenberg NS. Temporal crest canal: case report and statistics on a rare mandibular variant. Oral Surg Oral Med Oral Pathol 1986;62:10-2.

21. Kang JH, Lee KS, Oh MG, et al. The incidence and configuration of the bifid mandibular canal in Koreans by using cone-beam computed tomography. Imaging Sci Dent 2014;44:53-60.

22. Fukami K, Shiozaki K, Mishima A, et al. Bifid mandibular canal: confirmation of limited cone beam CT findings by gross anatomical and histological investigations. Dentomaxillofacial Radiol 2012;41:460-5.

23. Ferreira DCA, Fumes AC, Consolaro A, et al. Gubernacular cord and canal: does these anatomical structures play a role in dental eruption? Revista Sul-Brasileira de Odontologia (Online) 2013;10:167-71.

24. Hunter J. In: Johnson J, editor. The natural history of the human teeth: explaining their structure, use, formation, growth and diseases. 2nd ed 1778 .

25. Philipsen HP, Reichart PA. The development and fate of epithelial residues aftercompletion of the human odontogenesis with special reference to the origins of epithelial odontogenic neoplasms, hamartomas and cysts. Oral Biosci Med 2004;1:171-9.

26. Cahill DR, Marks Jr SC. Tooth eruption: evidence for the central role of the dental follicle. J Oral Pathol 1980;9:189-200. 